Supporting information

\title{
Scattering Fourier Transform Biosensor: Binary Mixture Consisting of Magnetic Ni Nanorings and Plasmonic $\mathrm{Au}$ Nanorods
}

\author{
Hajir Hilal ${ }^{a \dagger}$, Sungwoo Lee ${ }^{a \dagger}$, Insub Jung ${ }^{a, b}$, Sungjae Yoo ${ }^{a}$, and Sungho Park ${ }^{a^{*}}$
}

\begin{abstract}
aDepartment of Chemistry, Sungkyunkwan University, Suwon, 16419, South Korea
${ }^{\mathrm{b}}$ Current affiliation: Department of Chemistry, Northwestern University, Evanston, IL
\end{abstract}

*Email: spark72@skku.edu

These authors contributed equally 


\section{Table of contents}

Figure S1. UV-Vis spectra of shape evaluation

.S3

Figure S2. Histogram representing (A) size distribution of Au nanodisks (black line), Au@ Pt nanodisks (red line), Pt nanorings (blue line), and MN-rings (bluish-green line). S4

Figure S3. TEM images of various thicknesses of MN-rings . S5

Figure S4. Synthesis of Au@Pt@Ni nanospheres (MNSs) .56

Figure S5. Control experiment regarding magnetic rotational of different concentrations of MNrings. S7

Figure S6. FE-SEM images of various sizes of Au nanorods (Au NRs). S8

Figure S7. Optimization of conc. of Au NRs with $15 \mathrm{fM}$ of MN-rings in a binary mixture (sensing platform). .S9

Figure S8. Confirmation regarding surface modification of Au NRs.... S10 


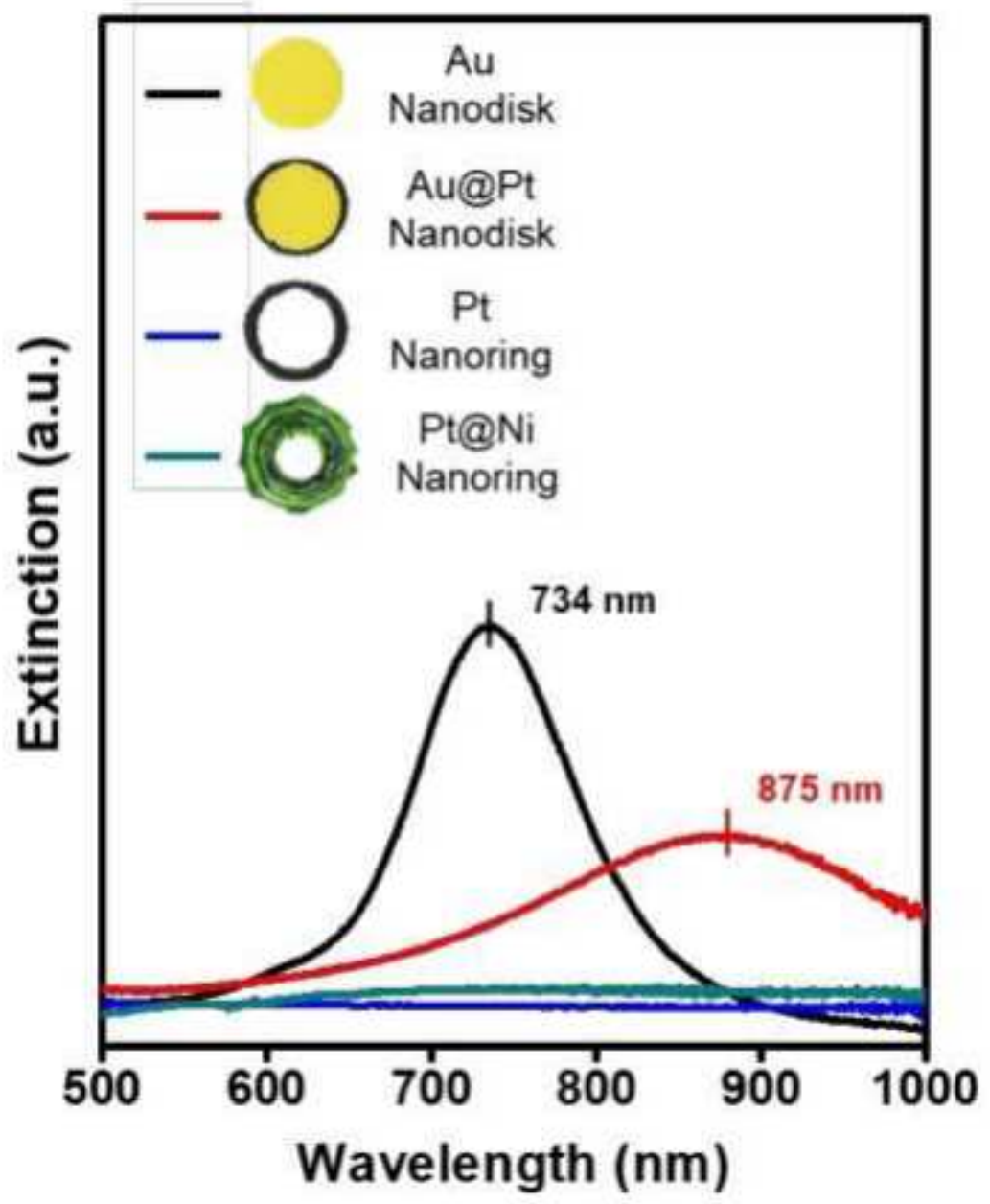

FigureS1.UV-Vis spectra of shape evaluation. Au nanodisks (black trace), Au@ Pt nanodisks (red trace), Pt nanorings (blue trace), Pt@ Ni nanorings (MN-rings) (bluish green trace). 

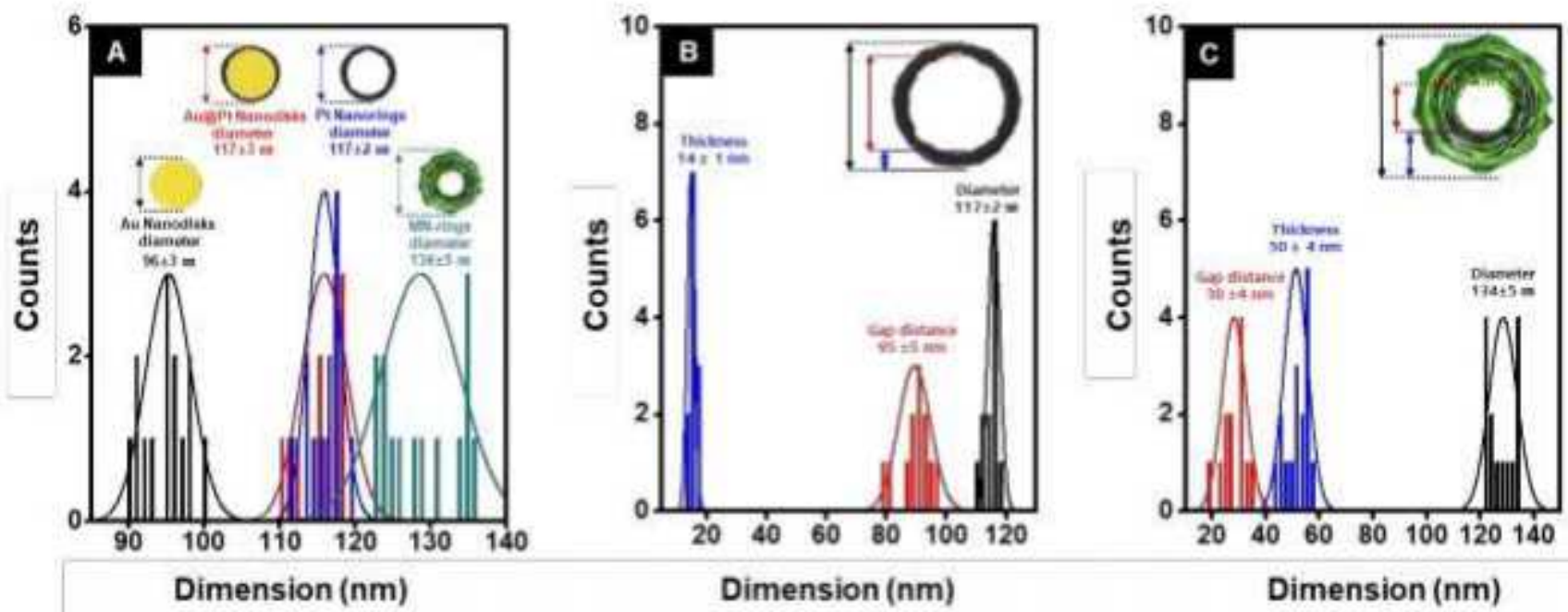

Figure S2. Histogram representing (A) size distribution of Au nanodisks (black line), Au@ Pt nanodisks (red line), Pt nanorings (blue line), and MN-rings (bluish-green line). (B-C) size distributions of $\mathrm{Pt}$ nanorings and $\mathrm{MN}$-rings including outer frame diameter (black line), gap distance (red line), and frame thickness (blue line). 

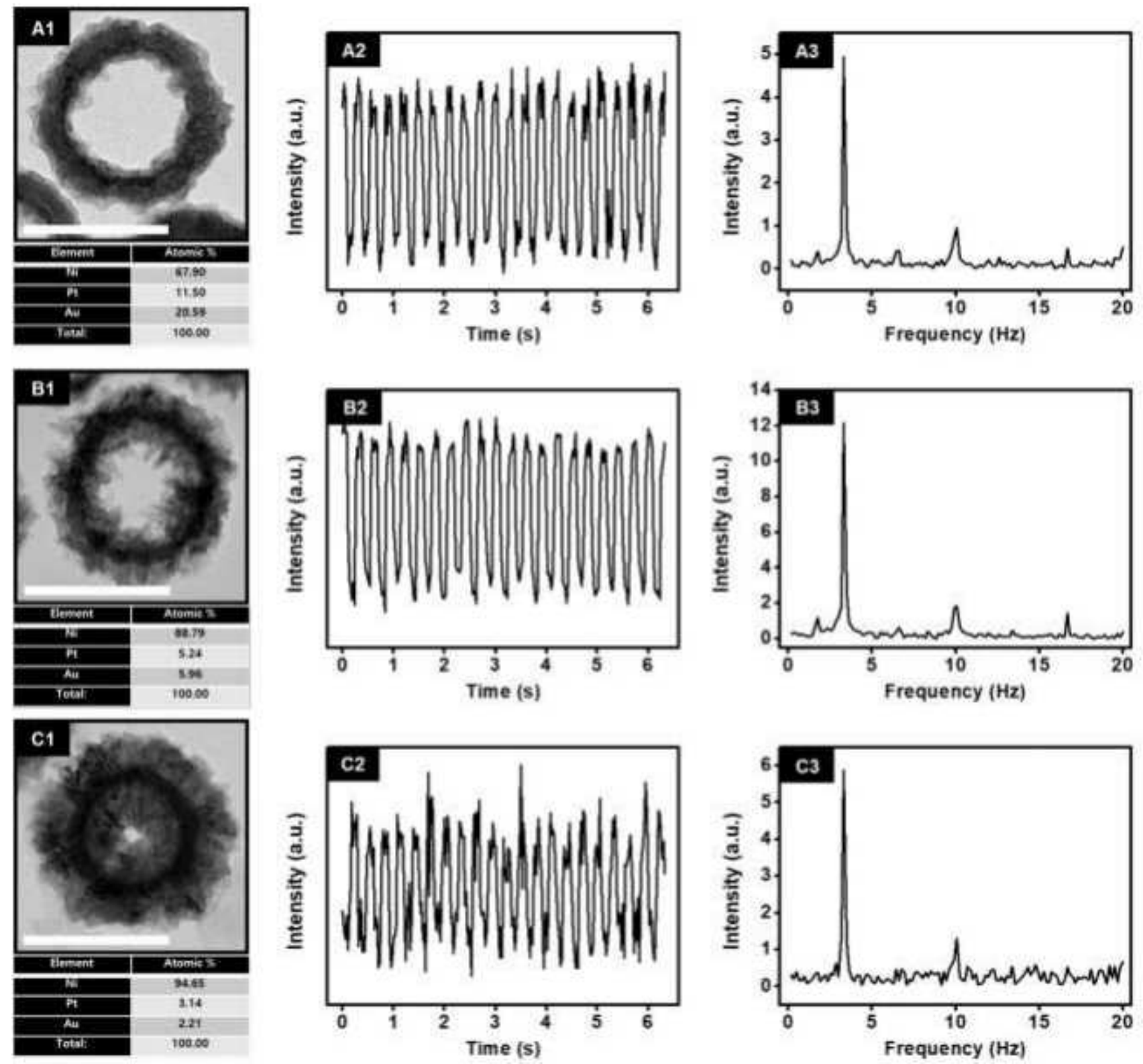

Figure S3. (A1, B1, C1) TEM images of various thicknesses of MN-rings. (A2, B2, C2) Optical responses of various thicknesses of $\mathrm{MN}$-rings under a rotating magnetic field $(\omega / 2 \pi=1.67 \mathrm{~Hz})$. (A3, B3, C3) Corresponding FFT plot of the optical response. These MN-rings were at the same concentration (measured by inductively coupled plasma atomic emission spectroscopy (ICP-AES)). 

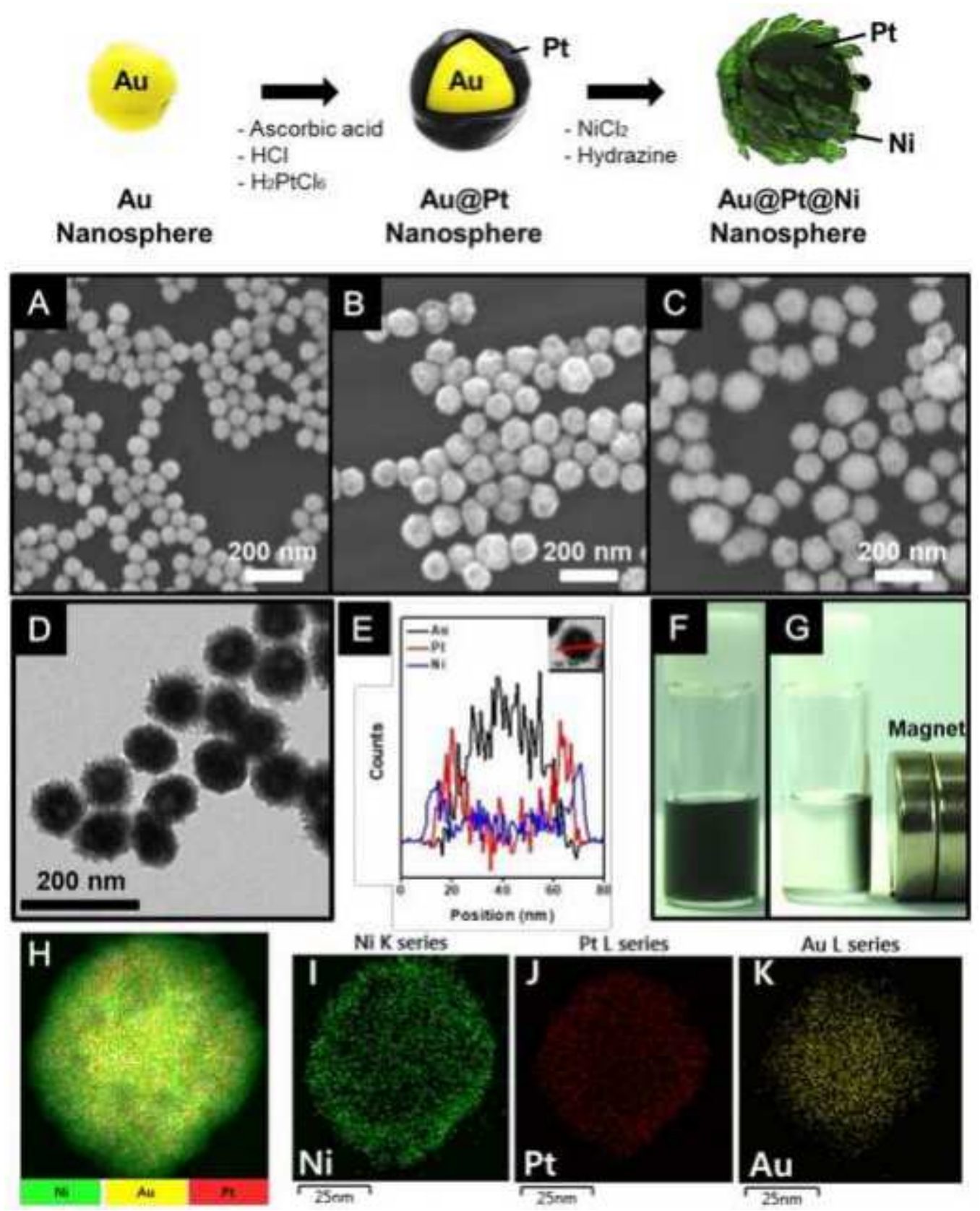

Figure S4. Synthesis of Au@Pt@Ni nanospheres (MNSs). Schematic illustration demonstrating synthetic pathway of MNSs. Au nanospheres (Au NSs) are used as starting material. Pt and Ni are deposited onto the Au NSs, consecutively. (A-C) FE-SEM images of Au NSs $(d=59 \pm 7 \mathrm{~nm})$, Au@Pt NSs $(d=91 \pm$ $12 \mathrm{~nm})$, and Ni MNSs $(d=112 \pm 14 \mathrm{~nm})$ were obtained to measure size and morphology of resulting nanoparticles synthesized through each synthetic step. (D) A HR-TEM image and (E) EDS line mapping of MNSs. The line mapping was conducted along the red line in inset image. (black line: Au; red line: Pt; blue line: Ni). (F) Optical photographs of MNSs dispersed in water after sonication and (G) adsorbed to the walls of a vial due to a permanent magnet. (H) Combined EDS elemental mapping showing distribution of $\mathrm{Ni}$ (green), $\mathrm{Au}$ (yellow), and $\mathrm{Pt}$ (red). (I-K) Individual EDS elemental mapping of $\mathrm{Ni}(\mathbf{I}), \mathrm{Pt}(\mathbf{J})$, and $\mathrm{Au}$ (K) in MNS. 

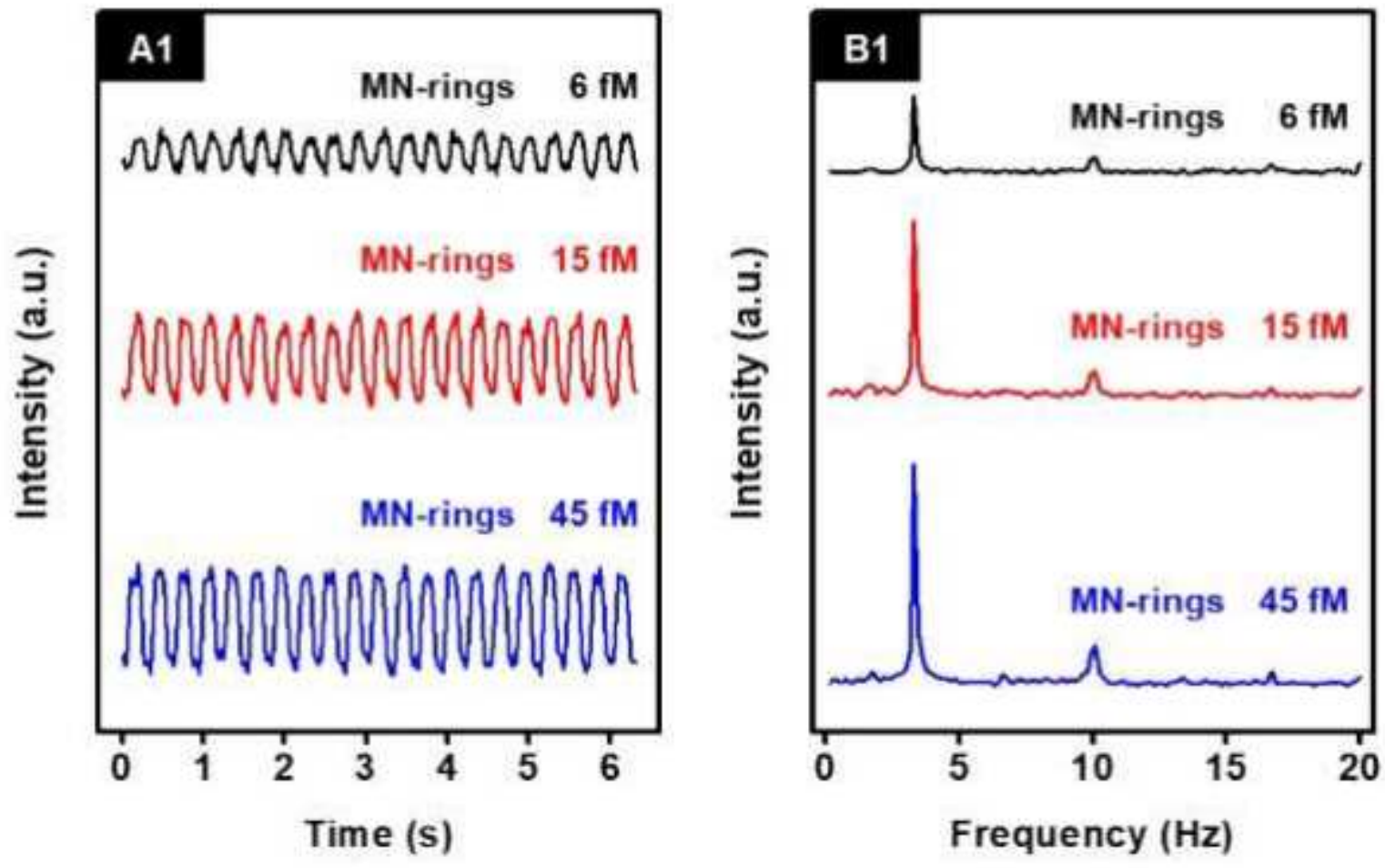

Figure S5. Control experiment regarding magnetic rotational of different concentrations of MNrings. (A) As a function of time intervals of $25 \mathrm{~ms}$. (B) Corresponding FFT plot of their optical responses. 

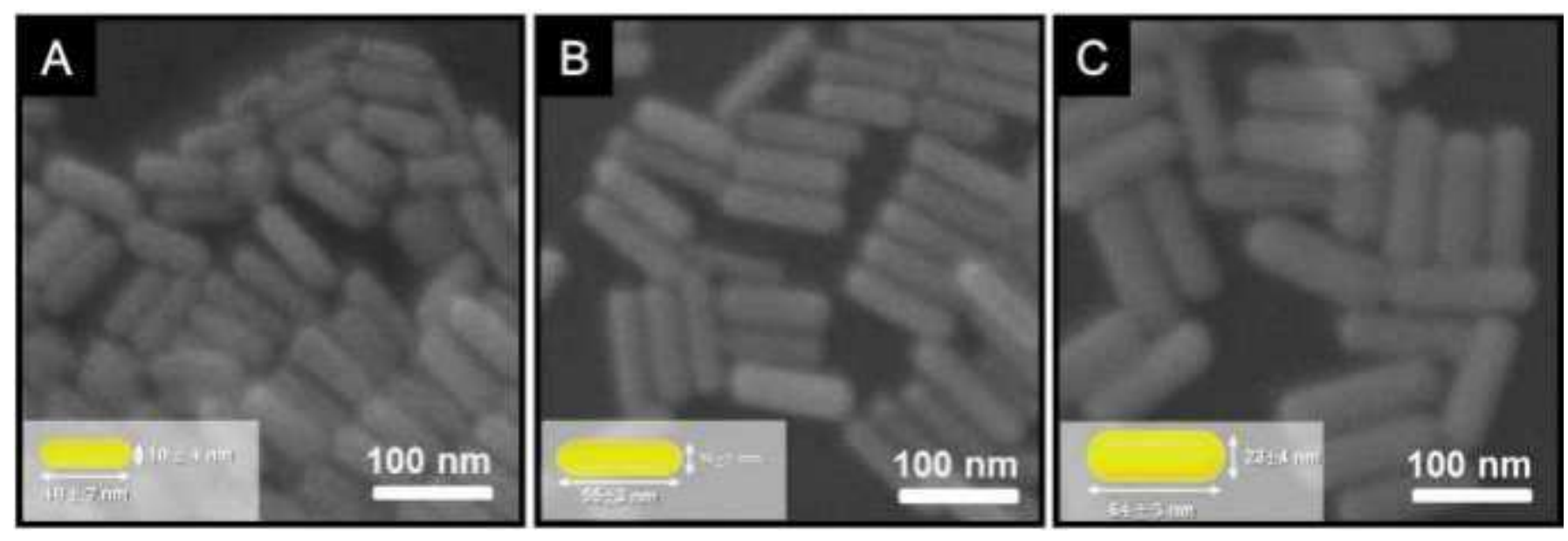

Figure S6. FE-SEM images of various sizes of Au nanorods (Au NRs). 

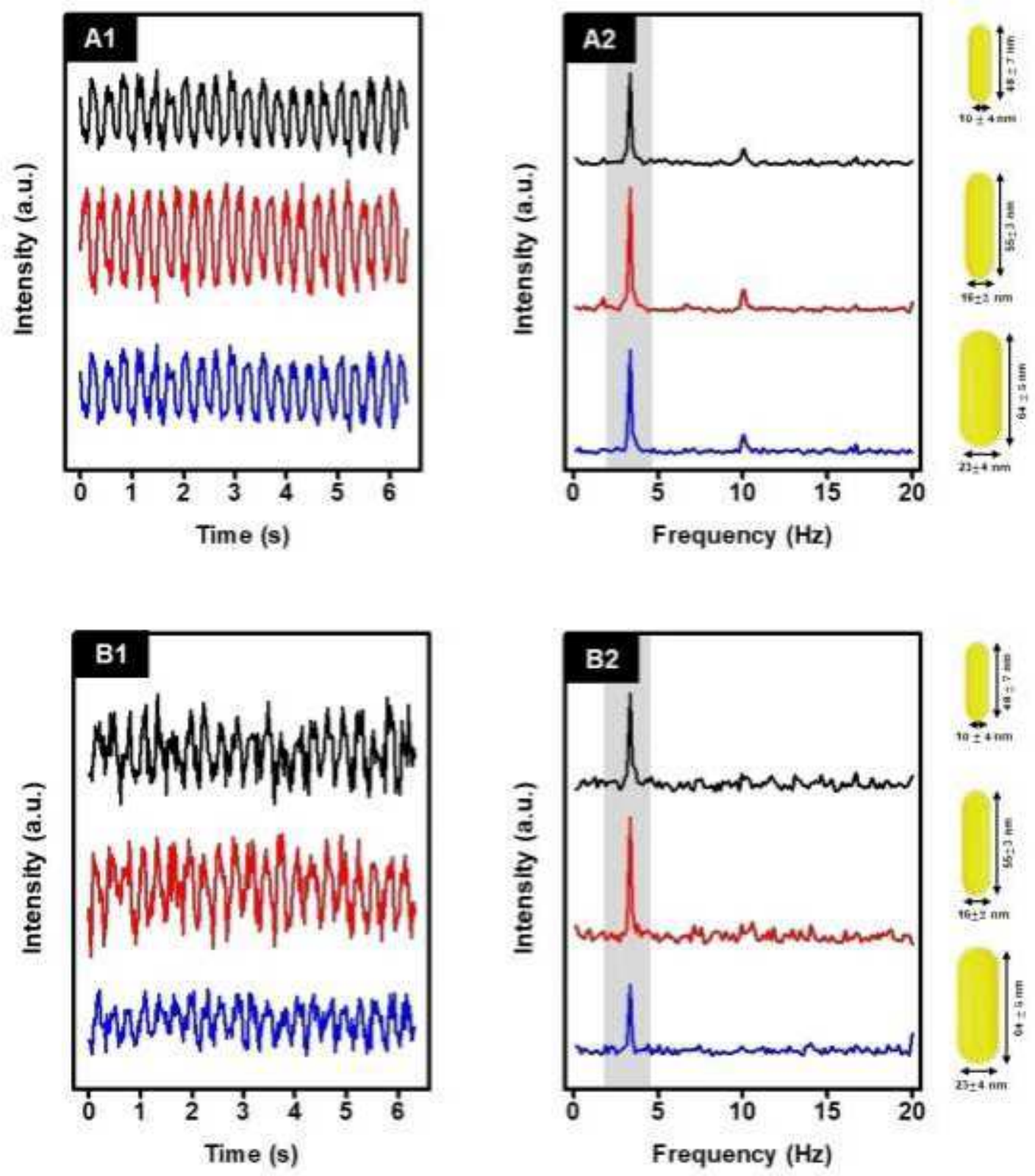

Figure S7. Optimization of conc. of Au NRs with $15 \mathrm{fM}$ of MN-rings in a binary mixture (sensing platform). $(\mathbf{A 1}, \mathbf{A 2}) 24 \mathrm{fM},(\mathbf{B 1}, \mathbf{B 2}) 60 \mathrm{fM}$ concentration of Au NRs 


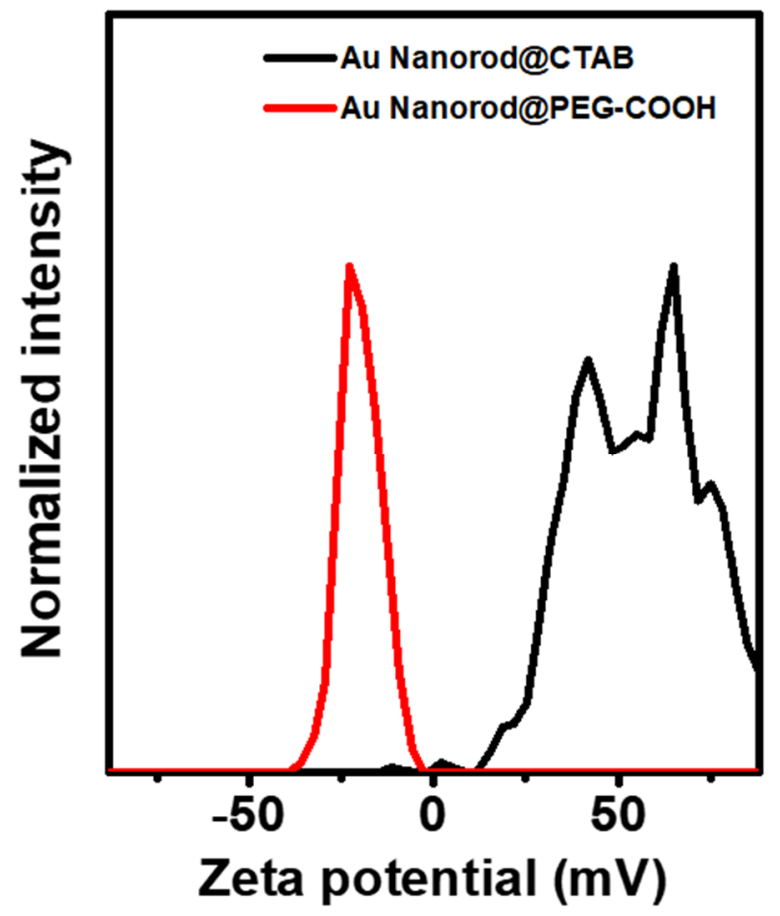

Figure S8. Confirmation regarding surface modification of Au NRs. Based on the measurement from DLS and UV-vis-NIR spectrophotometer, the surface of Au NRs was successfully modified with biomolecules. 